\title{
Comparison of Surrogate Measures for the Reliability and Redundancy of Water Distribution Systems
}

\author{
Tiku T. Tanyimboh ${ }^{1} \cdot$ Calvin Siew $^{1} \cdot$ Salah Saleh $^{1} \cdot$ \\ Anna Czajkowska ${ }^{1}$
}

(C) The Author(s) 2016. This article is published with open access at Springerlink.com

\begin{abstract}
An investigation into the effectiveness of surrogate measures for the hydraulic reliability and/or redundancy of water distribution systems is presented. The measures considered are statistical flow entropy, resilience index, network resilience and surplus power factor. Looped network designs that are maximally noncommittal to the surrogate reliability measures were considered. In other words, the networks were designed by multi-objective evolutionary optimization free of any influence from the surrogate measures. The designs were then assessed using each surrogate measure and two accurate but computationally intensive measures namely hydraulic reliability and pipe-failure tolerance. The results indicate that by utilising statistical flow entropy, the reliability of the network can be reasonably approximated, with substantial savings in computational effort. The results for the other surrogate measures were often inconsistent. Two networks in the literature were considered. One example involved a range of alternative network topologies. In the other example, based on wholelife cost accounting, alternative design and upgrading schemes for a 20-year design horizon were considered. Pressure-dependent hydraulic modelling was used to simulate pipe failures for the reliability calculations.
\end{abstract}

Keywords Pressure-dependent analysis $\cdot$ Hydraulic reliability and redundancy $\cdot$ Resilience index $\cdot$ Surplus power factor · Statistical flow entropy $\cdot$ Water distribution system

\section{Introduction}

The construction and subsequent rehabilitation and upgrading of a water distribution system represents a major economic infrastructure investment that necessitates an optimal long-term

Tiku T. Tanyimboh

tiku.tanyimboh@strath.ac.uk

1 Department of Civil and Environmental Engineering, University of Strathclyde Glasgow, James Weir Building, 75 Montrose Street, Glasgow G1 1XN, UK 
strategy. In the design of water distribution systems hydraulic reliability and failure tolerance are considered measures of robustness that determine the ability of the network to satisfy demands under both normal and abnormal operating conditions. However, reliability measures are particularly difficult to evaluate (Wagner et al. 1988). The traditional approach to achieving low-cost designs involves minimising the construction costs while satisfying the pressure requirements throughout the network. These designs aim to utilise the smallest pipe sizes with little or no spare capacity built-in to mitigate the effects of component failures or any increase in the demands. To improve the long-term performance of water distribution systems a more balanced approach that includes some measures of hydraulic reliability in the design process has been advocated (Templeman 1982). Various reliability measures for water distribution systems have been proposed previously. Measures such as hydraulic reliability and failure tolerance are so computationally demanding that their direct incorporation in optimization algorithms is often impractical. Several surrogate measures which sacrifice accuracy but offer a substantial reduction in the computational effort have therefore been suggested e.g. flow entropy, resilience index and surplus power factor.

For over two decades, flow entropy has been considered in the design of water distribution networks as it seems that an increase in the entropy corresponds generally to improvements in the hydraulic reliability (Awumah et al. 1991). It has been used also for layout optimization as it has the advantage that it reflects the arrangement of the paths in a distribution network (Tanyimboh and Sheahan 2002; Saleh and Tanyimboh 2014). The available evidence suggests that higher entropy values increase the uniformity of the pipe diameters along with the reliability (Tanyimboh and Templeman 1993b; Tanyimboh and Setiadi 2008). Strong positive correlation between flow entropy and both hydraulic reliability and failure tolerance has been reported (Tanyimboh and Templeman 2000; Tanyimboh et al. 2011; Gheisi and Naser 2015). Atkinson et al. (2014) observed that an increase in entropy promotes an increase in capacity that is more globally distributed throughout the distribution network. Recently, Liu et al. (2014) proposed an extension to the flow entropy function called diameter-sensitive flow entropy. Singh and Oh (2015) incorporated the Awumah et al. (1991) entropy formulation in a variant known as Tsallis entropy (Tsallis 1988). However Tanyimboh and Templeman (1993a, b, c, d) showed that the underlying entropy models by Awumah et al. (1991) do not satisfy certain axiomatic properties of probability schemes. Raad et al. (2010) also suggested an extension to the flow entropy function that includes the resilience index (Todini 2000).

One flow entropy value does not correspond to a unique design of a water distribution network due to the invariance of the entropy function; i.e. re-ordering the probabilities in a finite probability scheme leaves the statistical entropy value unchanged (Shannon 1948). Thus statistical entropy may be considered a dependable surrogate reliability measure only if different designs with similar entropy values possess comparable characteristics. It was discovered previously that in general designs with the same maximum entropy value are very similar in terms of hydraulic reliability and construction cost (Tanyimboh and Sheahan 2002; Tanyimboh and Setiadi 2008; Tanyimboh et al. 2011).

Comparisons of surrogate reliability measures include Prasad and Park (2004), Raad et al. (2010), Baños et al. (2011)), Tanyimboh et al. (2011), Wu et al. (2011), Greco et al. (2012), Atkinson et al. (2014), Liu et al. (2014) and Gheisi and Naser (2015). While some of the studies simulated operating conditions with insufficient pressure realistically with pressuredependent modelling (Liu et al. 2014; Gheisi and Naser 2015), others did not (e.g. Greco et al. 2012; Atkinson et al. 2014). It is well known that the demand-driven analysis approach often yields misleading results when applied to operating conditions with insufficient pressure (Tanyimboh et al. 1999, 2003). Tanyimboh et al. (1999) stated: "Compared to [demand-driven analysis] the results obtained by [head-driven analysis] are superior in the sense that, 
in addition to identifying precisely the nodes with inadequate flow/pressure the actual outflows at the nodes in question are determined. By contrast, the above does not hold true for [demand-driven analysis] (Tanyimboh and Tabesh 1997). In fact [demand-driven analysis] results can sometimes be both infeasible and very misleading."

Some of the studies (e.g. Prasad and Park 2004; Wu et al. 2011; Raad et al. 2010) did not include accurate measures of hydraulic reliability/redundancy. For example the pipe failure analysis in Raad et al. (2010) did not consider a probabilistic pipe failure model. Instead, it seems that pipe failures were taken as equally likely. However, it is generally accepted that large pipes fail less frequently than small pipes (Su et al. 1987; Cullinane et al. 1992; Khomsi et al. 1996). Also some studies (e.g. Raad et al. 2010) carried out comparisons on a consistent basis by optimizing each surrogate measure whereas other studies (e.g. Liu et al. 2014) optimized some but not all.

Water undertakings in the UK have statutory duties such as maintaining a satisfactory and continuous supply, and planned and unplanned interruptions are considered key performance indicators (see e.g. Twort et al. 2000, pp. 88 and 615-6). Consequently the performance of a water distribution network when one or more pipes are unavailable is an important design consideration. It has been suggested (see e.g. Tanyimboh and Templeman 1998; Kalungi and Tanyimboh 2003; Tanyimboh and Kalungi 2009) that the failure tolerance be considered in addition to the hydraulic reliability, as their mutual relationship is not monotonic. The reason is that water distribution networks are exceedingly complex systems. Investigations into surrogate reliability measures (e.g. Liu et al. 2014, Raad et al.) and the reliability of water distribution systems in general often do not address failure tolerance explicitly. It is worth clarifying that tolerance to component unavailability relates, in the first instance, primarily to the prescribed statutory demands rather than demand uncertainty.

The aim of this paper is to investigate the similarity of designs of water distribution networks whose maximum entropy values are equal, based on energy dissipation. If flow entropy is consistent, then such designs should have similar underlying characteristics. The paper also investigates the effectiveness of the resilience index, network resilience, surplus power factor and flow entropy, as surrogate measures of the reliability/redundancy of water distribution systems. Surrogate reliability measures are often maximised when used to design water distribution systems (Prasad and Park 2004; Baños et al. 2011; Wu et al. 2011; Raad et al. 2010; Farmani and Butler 2014; Liu et al. 2014; Saleh and Tanyimboh 2014). However, any surrogate measure that is optimized in the design process can be expected to have an unfair advantage. This is addressed by comparing the surrogate measures on an equal basis in this article. The influence of the network topology is considered also, together with the effects of pipe failures. Two networks in the literature are analysed and discussed.

\section{Hydraulic Reliability Measures}

Hydraulic reliability represents the probabilistic expectation of the ratio of the flow delivered to the flow required (Tanyimboh and Templeman 1995, 1998; Gargano and Pianese 2000; Tanyimboh et al. 2001). The failure tolerance, similarly, represents the probabilistic expectation of the ratio of the flow delivered to the flow required when one or more components of the distribution system are unavailable (Tanyimboh and Templeman 1995, 1998; Tanyimboh et al. 2001). The evaluation of the hydraulic reliability requires multiple analyses of the distribution system under both normal and subnormal operating conditions. This requires pressure-dependent modelling (Bhave 1981; 
Germanopoulos 1985; Gupta and Bhave 1996; Ackley et al. 2001). Pressure-dependent modelling differs from demand-driven modelling in that pressure-dependent modelling treats the available flow at a demand node as a function of the nodal pressure (e.g. Shirzad et al. 2013; Kovalenko et al.et al. 2014; Ciaponi et al. 2015; Abdy Sayyed et al. 2015) whereas demand-driven modelling equates the available nodal flow to the demand.

Pressure-dependent modelling was used here to simulate the post-failure pipe closures. Pipe closures reduce the flow carrying capacity of a water distribution system. Pressure-dependent modelling is therefore essential to ensure the simulations are realistic. Pressure- dependent modelling was carried out with the hydraulic models PRAAWDS (Tanyimboh et al. 2003; Tanyimboh and Templeman 2010) and EPANET-PDX (Siew and Tanyimboh 2012a; Seyoum et al. 2013; Seyoum and Tanyimboh 2014). It was assumed the pipes can be closed individually; in practice the actual locations of isolation valves would be considered. Only failures involving one pipe at a time were considered due to the low probability of multiple-pipe failures (Su et al. 1987; Tanyimboh and Templeman 1998; Gargano and Pianese 2000). We investigated several pipe failure models including: (a) Su et al. 1987; (b) Fujiwara and Tung 1991; (c) Cullinane et al. 1992; (d) Khomsi et al. 1996; and (e) Tabesh et al. 2009. Cullinane et al. (1992) and Khomsi et al. (1996) proved the most consistent. Both models gave very similar results and so only the results based on Cullinane et al. (1992) are included here.

\subsection{Statistical Entropy}

Informational entropy is a measure of the amount of uncertainty that a probability distribution represents (Shannon 1948). The flow entropy of a water distribution system is a measure of the relative uniformity of the pipe flow rates (Tanyimboh and Templeman 1993a, b, c, d).

$$
\frac{S}{K}=-\sum_{1=1}^{I} \frac{Q_{i}}{T} \ln \left(\frac{Q_{i}}{T}\right)-\frac{1}{T} \sum_{j=1}^{J} T_{j}\left[\frac{Q_{j}}{T_{j}} \ln \left(\frac{Q_{j}}{T_{j}}\right)+\sum_{i j \in N_{j}} \frac{q_{i j}}{T_{j}} \ln \left(\frac{q_{i j}}{T_{j}}\right)\right]
$$

where $S / K$ is the entropy with $K$ an arbitrary positive constant; $T$ is the total supply; $T_{j}$ is the total flow reaching node $j ; Q_{i}$ represents the inflow at a supply node; $Q_{j}$ represents the demand at a demand node; $q_{i j}$ is the flow rate in pipe $i j ; I$ represents the number of supply nodes; $J$ represents the number of demand nodes; and $N_{j}$ represents all the pipe flows from node $j$.

\subsection{Hydraulic Reliability}

Hydraulic reliability may be characterised as the extent to which the distribution system fulfills the demands at adequate pressure, considering both normal and abnormal operating conditions (Tanyimboh and Templeman 1995, 1998, 2000).

$$
\begin{aligned}
& R=\frac{1}{T}\left(p(0) T(0)+\sum_{m=1}^{M} p(m) T(m)+\sum_{m=1}^{M-1} \sum_{n=m+1}^{M} p(m, n) T(m, n)+\ldots\right) \\
& +\frac{1}{2}\left(1-p(0)-\sum_{m=1}^{M} p(m)-\sum_{m=1}^{M-1} \sum_{n=m+1}^{M} p(m, n)-\ldots\right)
\end{aligned}
$$

$R$ represents the hydraulic reliability that takes values from 0 to $1 ; M$ is the number of links (e.g. pipes, valves and pumps) in the network; $p(0)$ is the probability that no link is out of service; $p(m)$ is the probability that only link $m$ is not in service; $p(m, n)$ is the probability that 
only links $m$ and $n$ are not in service; $T(0), T(m)$ and $T(m, n)$ are, respectively, the total flow supplied with all links in service, only link $m$ unavailable and only links $m$ and $n$ unavailable; $T$ is the sum of the nodal demands. Equation 2 is clearly truncated; it includes additional terms involving three or more simultaneous link failures i.e. $p(l, m, n), p(k, l, m, n)$ and $T(l, m, n)$, $T(k, l, m, n)$, etc. with analogous definitions.

Equation 2 has two major components. The first component (that starts with ${ }^{1} /{ }_{T}$ ) corresponds to the reliability defined as the probabilistic expectation of the fraction of the demand satisfied. The second component (that starts with $1 / 2$ ) is a correction factor that compensates for the omission of some operating conditions that involve the failure of multiple pipes simultaneously (Tanyimboh and Sheahan 2002). The correction factor is a function of the pipe failure probabilities only - its evaluation does not require a hydraulic simulation model. Therefore, the correction factor can provide guidance on the number of simultaneous failures worth considering.

\subsection{Failure Tolerance}

The failure tolerance represents the probabilistic expectation of the fraction of the required flow that the distribution system satisfies at adequate pressure when one or more components are not in service (Tanyimboh and Templeman 1995, 1998; Tanyimboh et al. 2001).

$$
F T=\frac{R-p(0) T(0) / T}{1-p(0)}
$$

FT is the failure tolerance that takes values from 0 to 1 . The effect of the FT function in Eq. 3 is to modify the reliability by removing the contribution of the fully connected network condition, during which all pipes are in service. The contributions of all the states or conditions that correspond to single-link failures could be removed also, to obtain the failure tolerance when two links or more are out of service. The numerator and denominator in Eq. 3 would then have an additional term each, i.e. $-\sum_{m} p(m) T(m) / T$ and $-\sum_{m} p(m)$, respectively. Clearly, the values of all the terms used in the FT function are available in Eq. 2; thus more terms of higher order could be included in theory. In practice the computational demands often outweigh any benefits ( $\mathrm{Su}$ et al. 1987; Tanyimboh 1993, page 221). An extended discussion with additional examples and illustrations is available in Gheisi and Naser $(2013,2015)$. For the individual demand nodes Eqs. 2 and 3 would remain essentially the same and the respective nodal demands and available flows simply replace the total network demand and available flow. The formal derivation of Eq. 3 is available in Tanyimboh and Templeman (1998).

\subsection{Resilience Index}

Todini (2000) proposed the resilience index as a possible measure of redundancy defined as

$$
R I=\frac{\sum_{j=1}^{n_{d}} Q_{j}\left(H_{j}-H_{j}^{r e q}\right)}{\sum_{r=1}^{n_{r}} Q_{r} H_{r}+\sum_{i=1}^{n_{p}} \frac{P_{i}}{\gamma}-\sum_{j=1}^{n_{d}} Q_{j} H_{j}^{r e q}}
$$

$R I$ is the resilience index; for demand node $j, Q_{j}$ is the demand, $H_{j}$ is the head and $H_{j}^{r e q}$ is the head above which the demand is satisfied in full; $Q_{r}$ and $H_{r}$ are the water supply flow rate 
and head at reservoir $r$, respectively; $P_{i}$ is the power input at pump $i ; \gamma$ is the specific weight of water; $n_{d}, n_{r}$ and $n_{p}$ are the number of demand nodes, reservoirs and pumps, respectively. The total power input to the network may be accounted for by the energy losses in the flow through the network and the power that is available at the demand nodes. Equation 4 shows the resilience index is a measure of the available surplus power that, potentially, could be further dissipated by the flow through network in the event that extra stresses arise (e.g. due to an increase in demand).

\subsection{Network Resilience}

Prasad and Park (2004) modified the resilience index to reflect the relative sizes of the pipes meeting at a demand node. For demand node $j$, the uniformity coefficient $u_{j}$ was defined as the ratio of the mean diameter to the largest diameter. The network resilience $N R$ is thus

$$
N R=\frac{\sum_{j=1}^{n_{d}} u_{j} Q_{j}\left(H_{j}-H_{j}^{r e q}\right)}{\sum_{r=1}^{n_{r}} Q_{r} H_{r}+\sum_{i=1}^{n_{p}} \frac{P_{i}}{\gamma}-\sum_{j=1}^{n_{d}} Q_{j} H_{j}^{r e q}}
$$

\subsection{Surplus Power Factor}

Vaabel et al. (2006) introduced the surplus power factor as a measure of the spare hydraulic capacity in a pipe. It is based on the pipe flow rate and takes values between 0 and 1 (Eq. 6).

$$
s_{i j}=\frac{P_{i j}^{\max }-P_{i j}}{P_{i j}^{\max }} ; \quad \forall i j
$$

For pipe $i j, s_{i j}$ is the surplus power factor; $P_{i j}^{\max }$ and $P_{i j}$ are the maximum and available hydraulic power at the downstream end of the pipe, respectively. Both the minimum $s_{\min }$ and mean $E\left(s_{i j}\right)$ of the surplus power have been reported previously (e.g. Wu et al. 2011).

$$
s_{\min }=\min \left\{s_{i j}, \forall i j\right\} ; \quad E\left(s_{i j}\right)=\frac{1}{N_{I J}} \sum_{i j} s_{i j}
$$

where $N_{I J}$ is the number of pipes.

\subsection{Energy Dissipation}

Rowell and Barnes (1982) suggested that flow in a pipe could be characterized with reference to the energy dissipation rate. For a water distribution network the energy dissipation rate is $E=\rho g \sum_{i j \in I J} q_{i j} h_{i j}$

$E$ is the energy dissipation rate; $\rho$ is the density of water; $g$ is the acceleration due to gravity; for pipe $i j, q_{i j}$ is the flow rate and $h_{i j}$ is the head loss; and $I J$ includes all pipes in the network. 


\section{Results and Discussion}

\subsection{Example 1}

This example involves 137 minimum-cost maximum-entropy designs based on 65 layouts for the network in Fig. 1 introduced by Awumah et al. (1991). Initially Tanyimboh and Sheahan (2002) and Tanyimboh and Setiadi (2008) generated the designs to investigate flow entropy. Subsequently Tanyimboh et al. (2011) used the designs to investigate resilience index. The head at the supply node is $100 \mathrm{~m}$; demand node elevations are $0 \mathrm{~m}$; required residual head at demand nodes is $30 \mathrm{~m}$; pipes are $1000 \mathrm{~m}$ long; Hazen-Williams roughness coefficient is 130; pipe diameters are continuous in the range 100 to $600 \mathrm{~mm}$.

There are 60 designs with a unique maximum entropy value and 77 designs that have the same maximum entropy value with one or more designs. The 77 designs with the same maximum entropy value as one or more designs belong to 29 maximum-entropy groups (or sets) with two to six designs. The coefficient of variation of the energy dissipation rate was used to assess the similarity of the designs within each maximum-entropy set. The results were compared as follows.

- Group A: The set comprising the 77 designs with non-unique maximum entropy values.

- Group B: The full set of 137 designs.

- Group C: The set comprising the 60 designs with unique maximum entropy values.

Based on the number of members in the different maximum entropy sets, the weighted mean of the coefficient of variation was 0.0092 . The weighted mean represents the average similarity within individual maximum entropy sets. The arithmetic mean was 0.0090 ; while the weighted mean considers the number of members in each set, the arithmetic mean does not. The smallest coefficient of variation was 0.0002 and the largest was 0.0326 . The coefficient of variation for Group A, B and C were: 0.0282, 0.1496 and 0.2022, respectively. As percentages,

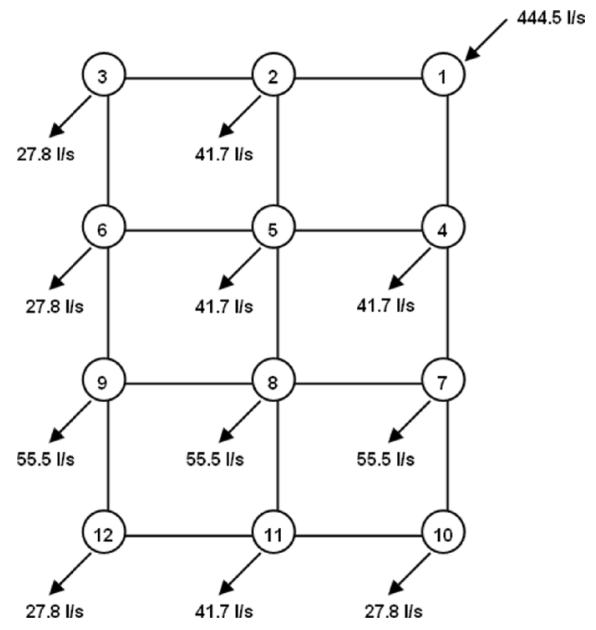

(a) Parent network

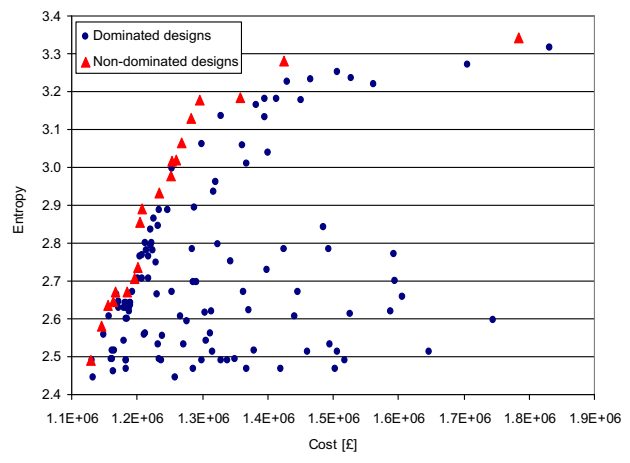

(b) Maximum entropy vs. cost

Fig. 1 Maximum entropy designs investigated in Example 1. a Parent network. b Maximum entropy vs. cost 
the ratio of the coefficient of variation for Group A, B and C to the weighted mean of the maximum entropy sets are $307 \%, 1,624 \%$, and $2,196 \%$, respectively. These results are consistent with the make-up of Group A, B and C. Group A consists of 29 clusters. Group $\mathrm{C}$ has no such clusters. Group B is a simple combination of Group A and C and is thus intermediate. The logical inference is that the designs within the maximum entropy sets have similar physical characteristics. This is also consistent with previous results (Tanyimboh and Sheahan 2002; Tanyimboh and Setiadi 2008; Tanyimboh et al. 2011) and supports the hypothesis that generally flow entropy is reasonably consistent.

Figure $1 \mathrm{~b}$ shows that 20 designs are non-dominated based on cost minimization and entropy maximization. The investigation of the surrogate reliability measures that follows is based on the 20 non-dominated designs. The hydraulic simulations were carried out with PRAAWDS (Program for Realistic Analysis of Availability of Water in Distribution Systems) (Tanyimboh et al. 2003; Tanyimboh and Templeman 2010). The pipe failure rates were estimated as in Cullinane et al. (1992). Table 1 shows positive correlation between flow entropy and both hydraulic reliability and failure tolerance. The correlation here based on the non-dominated solutions is greater than reported previously in Tanyimboh et al. (2011) for the full set of 137 solutions.

Table 1 also suggests there is no reasonable correlation between resilience index and both hydraulic reliability and failure tolerance. Queries about the resilience index have been discussed previously (e.g. Reca et al. 2008, Tanyimboh et al. 2008, 2011 and Farmani and Butler 2014). The network resilience, similarly, shows no clear trend. The modified resilience index by Jayaram and Srinivasan (2008) was not investigated as it is essentially the same as the resilience index for single-source networks (Tanyimboh et al. 2011). There is positive correlation for the average surplus power factor. As with flow entropy, the correlation is stronger with failure tolerance than reliability.

Table 1 Relationship between reliability and surrogate reliability measures

\begin{tabular}{|c|c|c|c|c|c|c|}
\hline \multirow{3}{*}{$\begin{array}{l}\text { Surrogate reliability } \\
\text { measures }\end{array}$} & \multicolumn{6}{|c|}{ Coefficient of determination $R^{2}$} \\
\hline & \multicolumn{2}{|l|}{ Example $1^{\mathrm{a}}$} & \multicolumn{2}{|l|}{ Example 2} & \multicolumn{2}{|l|}{ Example 3} \\
\hline & Reliability & $\begin{array}{l}\text { Failure } \\
\text { tolerance }\end{array}$ & Reliability & $\begin{array}{l}\text { Failure } \\
\text { tolerance }\end{array}$ & Reliability & $\begin{array}{l}\text { Failure } \\
\text { tolerance }\end{array}$ \\
\hline Flow entropy & $\begin{array}{l}0.64 \\
(0.522)\end{array}$ & $\begin{array}{l}0.83 \\
(0.803)\end{array}$ & 0.358 & 0.548 & 0.549 & 0.352 \\
\hline Resilience index & $\begin{array}{l}0.000 \\
(0.077)\end{array}$ & $\begin{array}{l}0.000 \\
(-0.002)\end{array}$ & -0.019 & -0.290 & 0.008 & 0.017 \\
\hline Network resilience & $\begin{array}{l}-0.003 \\
(-0.002)\end{array}$ & $\begin{array}{l}-0.017 \\
(-0.172)\end{array}$ & -0.130 & -0.501 & $\mathrm{U}^{\mathrm{b}}$ & $\mathrm{U}$ \\
\hline Mean surplus power factor & $\begin{array}{l}0.388^{\mathrm{c}} \\
\left(U^{b}\right)\end{array}$ & $\begin{array}{l}0.578^{\mathrm{c}} \\
(U)\end{array}$ & -0.125 & -0.038 & $\mathrm{U}$ & $\mathrm{U}$ \\
\hline Failure tolerance & $\begin{array}{l}0.73 \\
(0.707)\end{array}$ & - & 0.559 & - & 0.866 & - \\
\hline
\end{tabular}

${ }^{a}$ Corresponding values in Tanyimboh et al. (2011) are shown italicised in parentheses

${ }^{\mathrm{b}}$ Unavailable

${ }^{\mathrm{c}}$ For the minimum surplus power factor, $R^{2}$ values were -0.0158 and -0.0596 for reliability and failure tolerance, respectively 


\subsection{Example 2}

Surrogate reliability measures are often employed as objectives in the design optimization of water distribution systems. A query that arises is that, with a particular surrogate measure involved in the design process, the designs achieved may favour that particular measure. Therefore, the designs investigated next were optimized without reference to any surrogate measure. The assessment involves 32 minimum-cost designs for 32 new layouts for the network in Fig. 1. Continuous pipe diameters were adopted to avoid any surplus flow capacity in the network that may be unavoidable with discrete pipe sizes. This further ensures that subsequent comparisons of the designs and surrogate measures are robust and fair. The two parent layouts shown in Fig. 2 have a combined total of 32 fully-looped layouts that have not been investigated previously. Both Layout A and B will remain fully-looped if pipe 2-5 is removed or closed. Therefore, with four such "non-essential" pipes the total number of fullylooped layouts is 32 or $2 \times \sum_{i=0}^{i=4} C_{i}$.

The 32 new minimum-cost designs were generated with a multiobjective genetic algorithm NSGA II (Deb et al. 2002). Two objectives that were both minimized were used namely the construction cost and sum of the demand node pressure deficits. The minimum node pressure constraints were thus satisfied. EPANET 2 (Rossman 2002) was used for the hydraulic simulations that ensured the conservation of mass and energy constraints were satisfied. The decision variables were the pipe diameters. The cheapest feasible designs achieved are shown in Table 2. The costs vary from $£ 1,062,034$ to $£ 1,373,739$ and the maximum surplus head is $10 \mathrm{~mm}$. Any small surplus in the head at the critical node resulted mainly from rounding off the diameters. The critical node is the node that has the smallest residual head. The optimization was executed 10 times for each layout. Table 1 shows that only flow entropy has a reasonable correlation with hydraulic reliability and failure tolerance. These results may be indicative of a fundamental relationship between flow entropy and hydraulic reliability/ redundancy as the surrogate measures had no role in the pipe sizing. Pressure-dependent modelling in PRAAWDS was used for the reliability calculations with pipe failure rates estimated as in Cullinane et al. (1992).

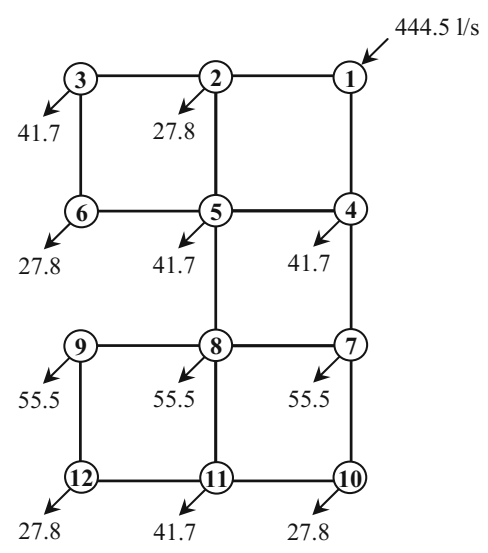

(a) Layout $\mathrm{A}$

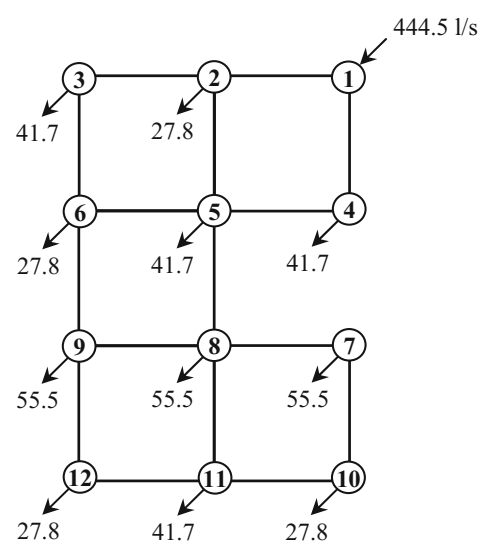

(b) Layout B

Fig. 2 Parent layouts for Example 2. a Layout A. b Layout B 


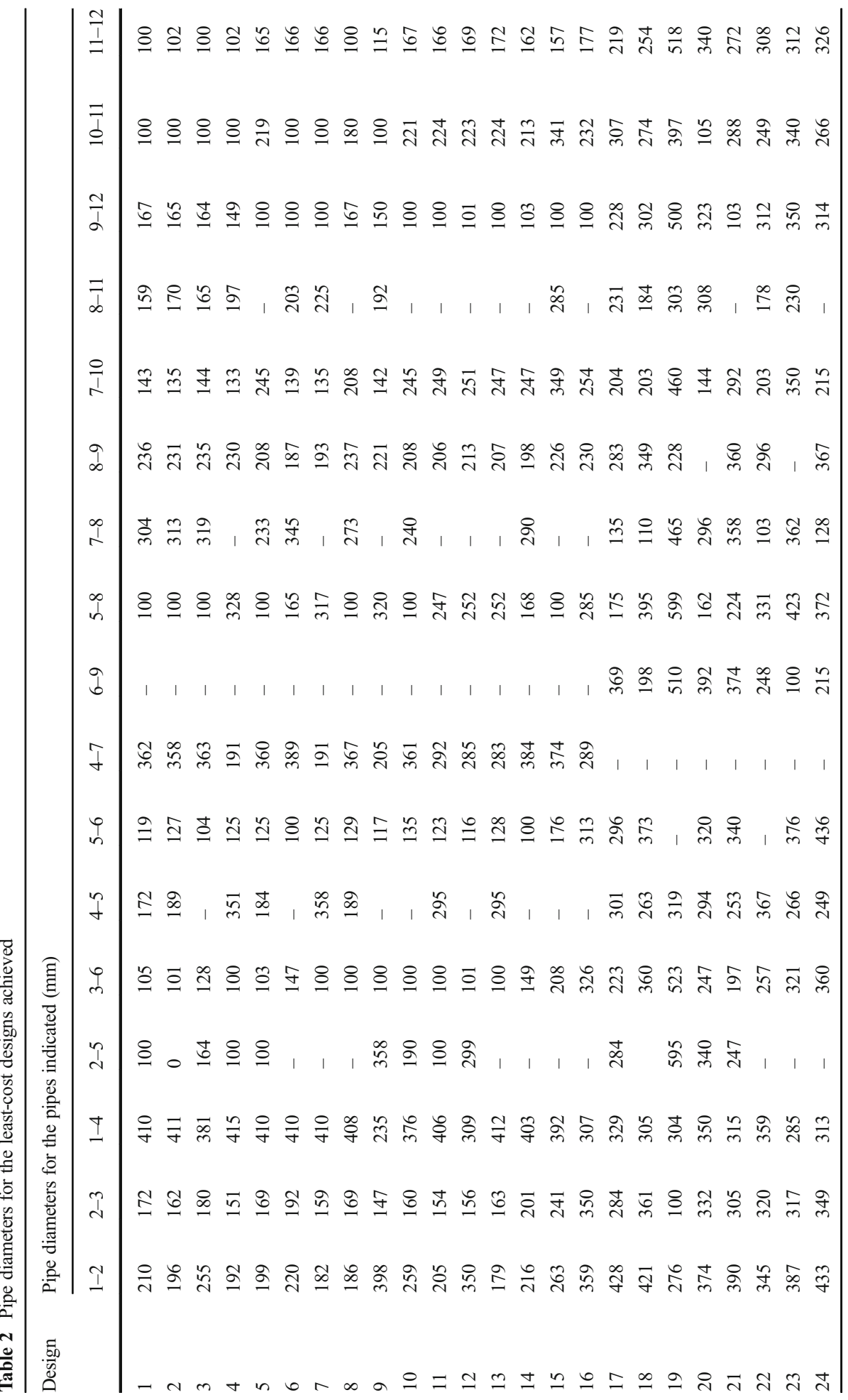




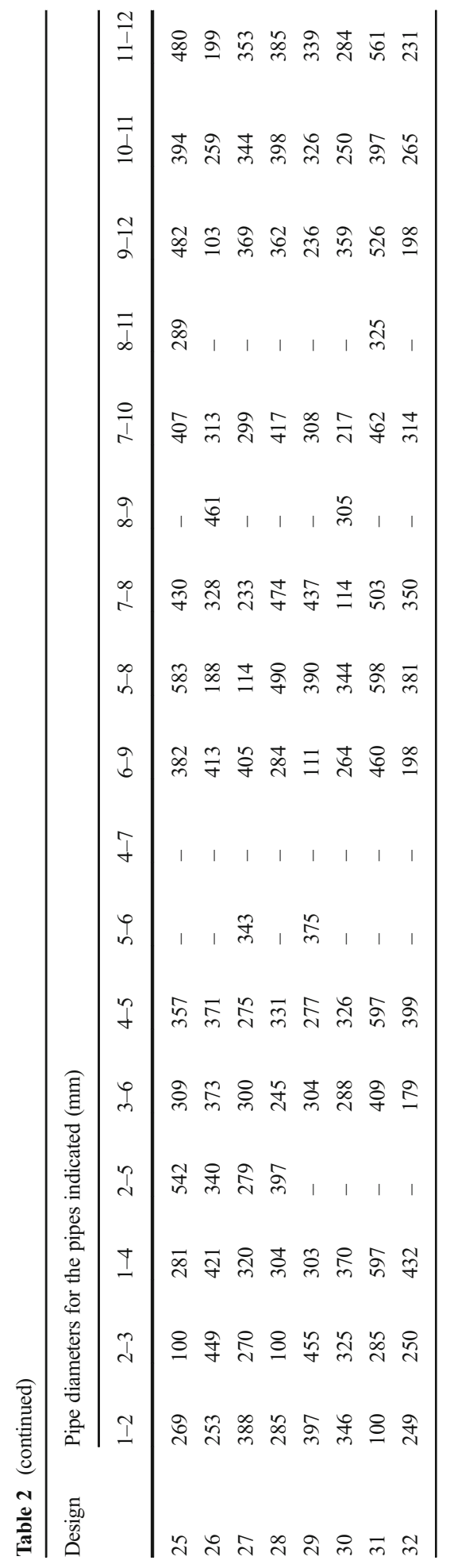




\subsection{Example 3}

The skeletonised network consists of a reservoir, 17 nodes and 21 pipes serving a population of 10,640 (Fig. 3a) (Tanyimboh and Kalungi 2008, 2009). The design optimization problem considers the deterioration over time of the structural integrity and hydraulic capacity of the pipes. The whole-life cost accounting method considers the discount and inflation rates. The costs include construction; repairs; and pipe failures and associated third party costs. The aim of the design is to minimize the total cost while satisfying the demands for a design horizon of 20 years. The decision variables are the pipe diameters, the rehabilitation and upgrading options i.e. paralleling and/or replacement, and the time of upgrading. A two-phase design with eight alternative Phase I durations of 7 to 14 years was considered. A complete description of the optimization problem is available in Tanyimboh and Kalungi $(2008,2009)$.

A penalty-free multi-objective evolutionary algorithm (PF-MOEA) was used to solve the optimization problem (Siew et al. 2014). The algorithm uses binary coding, single-bit mutation, single-point crossover and binary tournament selection for crossover. Ten optimization runs with random initial populations were carried out with 160,000 hydraulic simulations per optimization run. The population size was 100 . The probability of crossover and mutation were 1.0 and 0.005 , respectively. The minimum node pressure requirements were addressed

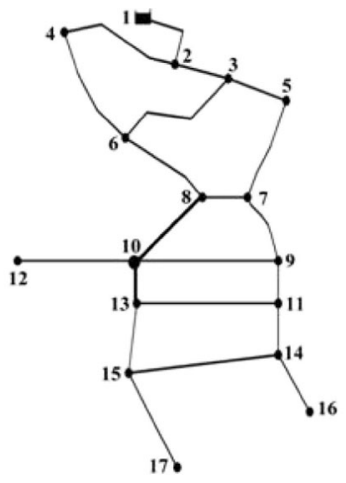

(a) Network layout

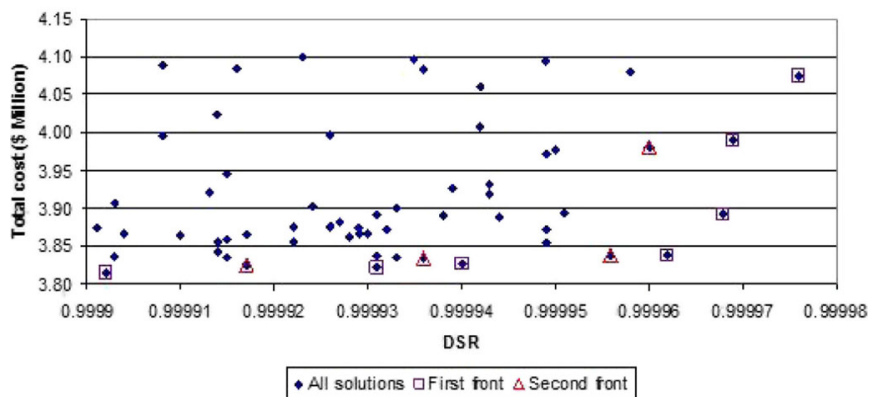

(b) Cost-effectiveness chart. DSR i.e. demand satisfaction ratio is the fraction of the demand that is satisfied

Fig. 3 Minimum-cost designs investigated in Example 3. a Network layout. b Cost-effectiveness chart. DSR i.e. demand satisfaction ratio is the fraction of the demand that is satisfied 
with pressure-dependent modelling in EPANET-PDX (pressure-dependent extension) (Siew and Tanyimboh 2012a, 2012b; Seyoum and Tanyimboh 2014). A desktop computer (with CPU of $3.2 \mathrm{~Hz}$ and RAM of $2 \mathrm{~GB}$ ) was used. The average CPU time per optimization run was $2.58 \mathrm{~h}$. Details of the methodology and algorithm are available in Siew et al. (2014) and Siew and Tanyimboh (2012a, b). The optimization algorithm (PF-MOEA) has consistently achieved good results previously. EPANET-PDX was used for the pipe failure simulations in the reliability calculations and pipe failure rates were estimated as in Cullinane et al. (1992).

Table 3 presents the total costs for the solutions achieved by the genetic algorithm. The linear programming (LP) solutions by Tanyimboh and Kalungi $(2008,2009)$ are included for comparison purposes. All the solutions shown satisfy the demands in full. The reliability values considered here relate to the network and operating conditions at the end of the design horizon i.e. Year 20. The cheapest solution achieved by the evolutionary algorithm (EA) costs $\$ 3,814,298$, for a Phase I duration of 9 years. The cheapest linear programming (LP) solution costs $\$ 3,953,663$, for a Phase I duration of 11 years. It can be observed that apart from the solution with a Phase I duration of 14 years, the EA solutions are cheaper than the corresponding LP solutions. The main reason is that the LP solutions are maximum-entropy designs that are inherently more expensive (Tanyimboh and Templeman 2000).

Each execution of the evolutionary algorithm generates designs for eight alternative Phase I duration of 7 to 14 years. Selecting the cheapest feasible solution from each Phase I duration provides in total 80 solutions from 10 optimization runs. In practice and as might be expected there are fewer than 80 solutions as some of the solutions feature in multiple runs. The overall best solutions were identified as shown in Fig. $3 b$ based on the cost effectiveness of the solutions. All the solutions are feasible; however the maximum difference in cost between the solutions is approximately $\$ 300,000$. Solutions in the two leading non-dominated fronts in Fig. $3 b$ were selected for further analyses; two fronts were necessary as the first front has only seven solutions. Then the correlation analysis was carried out for resilience index and flow entropy that are used the most. Table 1 shows that the correlation between flow entropy and both reliability and failure tolerance is reasonable while the relationship between resilience index and both reliability and failure tolerance is much weaker.

Table 3 Optimal costs for the design and capacity expansion schemes achieved

\begin{tabular}{|c|c|c|c|c|c|c|c|}
\hline \multirow{2}{*}{$\begin{array}{l}\text { Phase I Durations } \\
\text { (years) }\end{array}$} & \multirow{2}{*}{$\begin{array}{l}\text { Phase II Durations } \\
\text { (years) }\end{array}$} & \multicolumn{2}{|c|}{ PF-MOEA Costs (\$ Million) } & \multicolumn{4}{|c|}{${ }^{\mathrm{a}}$ LP Costs (\$ Million) } \\
\hline & & Phase I & Phase II & Total & Phase I & Phase II & Total \\
\hline 7 & 13 & 2.862 & 0.998 & 3.860 & 2.907 & 1.386 & 4.293 \\
\hline 8 & 12 & 2.950 & 0.877 & 3.827 & 3.006 & 1.071 & 4.077 \\
\hline 9 & 11 & 3.047 & 0.768 & $3.814^{\mathrm{b}}$ & 3.084 & 0.966 & 4.050 \\
\hline 10 & 10 & 3.148 & 0.689 & 3.837 & 3.200 & 0.789 & 3.989 \\
\hline 11 & 9 & 3.281 & 0.593 & 3.873 & 3.315 & 0.639 & $3.954^{\mathrm{b}}$ \\
\hline 12 & 8 & 3.399 & 0.504 & 3.902 & 3.414 & 0.544 & 3.958 \\
\hline 13 & 7 & 3.565 & 0.415 & 3.980 & 3.523 & 0.461 & 3.984 \\
\hline 14 & 6 & 3.725 & 0.334 & 4.059 & 3.631 & 0.409 & 4.040 \\
\hline
\end{tabular}

\footnotetext{
a The linear programming (LP) costs are from Tanyimboh and Kalungi (2008)

${ }^{\mathrm{b}}$ The cheapest solution for each approach is highlighted in bold
} 
To demonstrate further the advantages of entropy as a surrogate performance measure, Fig. 4a shows a plot of the cost against entropy. The range of entropy values is narrow and the

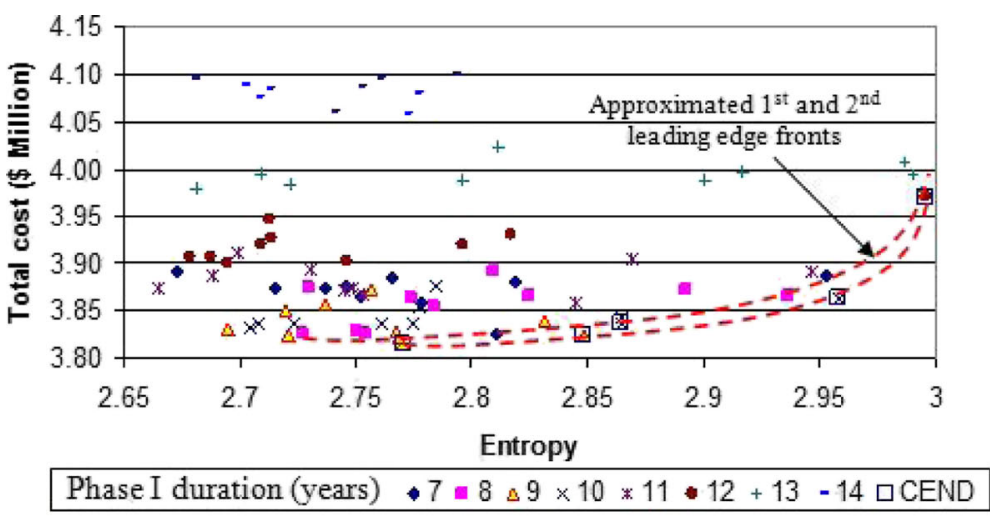

(a) Flow entropy

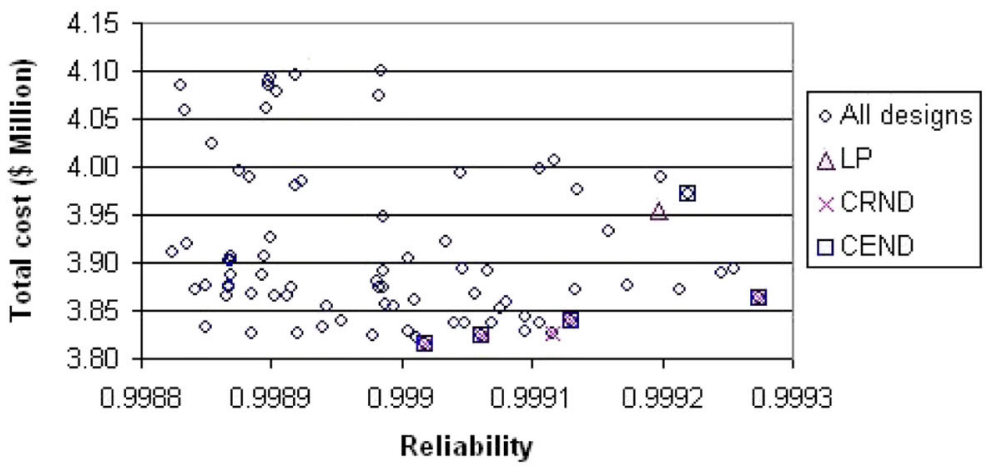

(b) Reliability

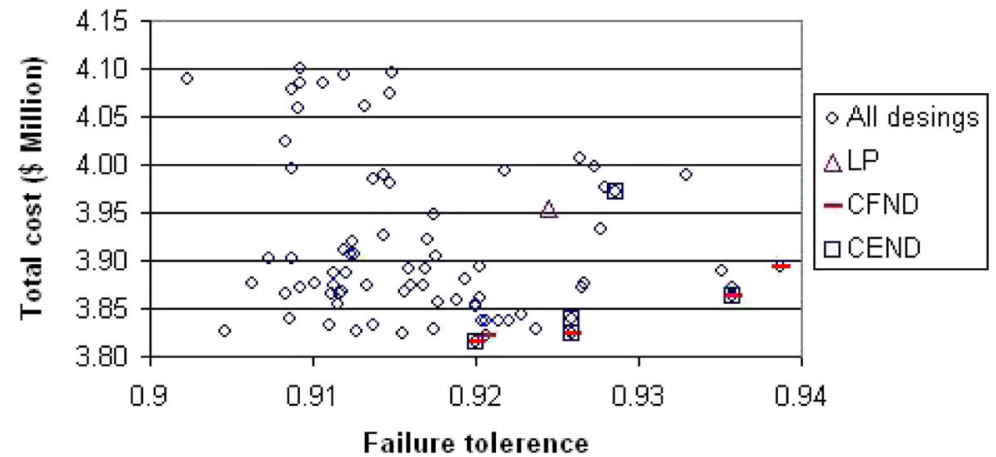

(c) Failure tolerance

Fig. 4 Reliability and failure tolerance cross-checks for the cost-entropy nondominated solutions. CEND, CFND and CRND are respectively: cost-vs-entropy, cost-vs-failure tolerance and cost-vs-reliability nondominated solutions. a Flow entropy. b Reliability. c Failure tolerance 
reason is that entropy was not considered in the design optimization. Due to the narrow range of entropy values, there are only five non-dominated solutions in the cost-entropy space. Also, it can be seen that the solutions with Phase I durations of 12 to 14 years tend to be uncompetitive. Figure $4 \mathrm{~b}$ and $\mathrm{c}$ show plots of cost vs. reliability and cost vs. failure tolerance, respectively. The best linear programming solution is shown also. The aim here is to illustrate the efficiency and robustness of screening the solutions to be considered further using entropy. Figure $4 \mathrm{~b}$ shows that five solutions are non-dominated in the cost-reliability space of which four are also non-dominated in the entropy-cost space. Figure $4 \mathrm{c}$ shows that five solutions are non-dominated in the cost-failure tolerance space of which three are also non-dominated in the entropy-cost space. Therefore, it can be inferred that a solution that is Pareto-optimal in the entropy-cost space will likely perform well based on reliable and/or failure tolerance (Figure $4 \mathrm{~b}$ and c). Also, it can be seen that the Pareto-optimal front in the entropy-cost space also approximates well the Pareto-optimal fronts in the reliability-cost and failure tolerancecost spaces. It is worth noting that the solutions in the reliability-cost and failure tolerance-cost fronts that are not in the first entropy-cost front are in fact in the second entropy-cost front.

Considering cost, hydraulic reliability and failure tolerance, three solutions are nondominated. These solutions can be identified in Fig. $4 \mathrm{c}$ as the three nondominated solutions that are also nondominated in the entropy-cost space. The pipe diameters and other details of the solutions are available in Siew et al. (2014). Multiobjective evolutionary algorithms often yield vast populations of candidate solutions. These results show that it is highly effective to use entropy to select the solutions that merit further consideration.

\section{Conclusions}

A comparison of surrogate reliability and redundancy measures for water distribution systems has been carried out. The measures considered were flow entropy, resilience index, network resilience and surplus power factor. These measures were assessed considering single-pipe failures for two networks in the literature. Both pre-existing maximum entropy designs in the literature and new least cost designs were investigated. The main conclusion is that only flow entropy seems to have the properties of a reasonable measure of hydraulic reliability and redundancy for water distribution systems. Three cases were considered and only flow entropy had a positive correlation with both hydraulic reliability and redundancy consistently. The other measures often had negative or very little correlation. It was also shown that generally designs with the same maximum entropy value tend to have comparable characteristics. These results seem to reinforce the hypothesis that the relationship between flow entropy and hydraulic reliability/redundancy is fundamental.

Some limitations of the study are that the flow entropy model investigated was developed for a single operating condition. It would be desirable to investigate possible extensions to multiple operating conditions (Czajkowska and Tanyimboh 2013). For example, if only one operating condition based on the peak flow is used to optimize the design then in general the system may be infeasible for other critical operating conditions (Alperovits and Shamir 1977). More research is thus indicated including comparisons based on large networks.

Acknowledgments This research was funded in part by the UK Engineering and Physical Sciences Research Council (EPSRC Grant Reference EP/G055564/1), the Government of Libya, the UK Government (Universities 
UK Overseas Research Students' Award Scheme) and the University of Strathclyde. We acknowledge with thanks the contribution of Douglas Bale (EPSRC Vacation Bursaries, 2010; EPSRC Reference EP/P502764/1).

All supporting data are included in the manuscript.

\section{Compliance with Ethical Standards}

Conflict of Interest There is no conflict of interest.

Open Access This article is distributed under the terms of the Creative Commons Attribution 4.0 International License (http://creativecommons.org/licenses/by/4.0/), which permits unrestricted use, distribution, and reproduction in any medium, provided you give appropriate credit to the original author(s) and the source, provide a link to the Creative Commons license, and indicate if changes were made.

\section{References}

Abdy Sayyed MAH, Gupta R, Tanyimboh TT (2015) Noniterative application of EPANET for pressure dependent modelling of water distribution systems. Water Resour Manag 29:3227-3242

Ackley JRL, Tanyimboh TT, Tahar B, Templeman AB (2001) Head-driven analysis of water distribution systems. In: Ulanicki et al (eds) Water software systems: theory and applications, vol 1. Research Studies Press, England, pp 183-192. ISBN 0863802745

Alperovits E, Shamir U (1977) Design of optimal water distribution systems. Water Resour Res 13(6):885-900

Atkinson S, Farmani R, Memon FA, Butler D (2014) Reliability indicators for water distribution system design: comparison. J Water Resour Plann Manag 140(2):160-168

Awumah K, Goulter I, Bhatt SK (1991) Entropy based redundancy measures in water distribution networks. J Hydraul Eng 117(5):595-613

Baños R, Reca J, Martínez J, Gil C, Márquez AL (2011) Resilience indexes for water distribution network design: a performance analysis under demand uncertainty. Water Resour Manag 25:2351-2366

Bhave PR (1981) Node flow analysis of water distribution systems. J Transp Eng 107(4):457-467

Ciaponi C, Franchioli L, Murari E, Papiri S (2015) Procedure for defining a pressure-outflow relationship regarding indoor demands in pressure-driven analysis of water distribution networks. Water Resour Manag 29:817-832

Cullinane MJ, Lansey KE, Mays LW (1992) Optimization-availability-based design of water distribution networks. J Hydraul Eng 118(3):420-441

Czajkowska AG, Tanyimboh TT (2013) Water distribution network optimization using maximum entropy and multiple loading patterns. Water Sci Technol Water Supply 13(5):1265-1271

Deb K, Pratap A, Agarwal S, Meyarivan T (2002) A fast and elitist multiobjective genetic algorithm: NSGA-II. IEEE Trans Evol Comput 6(2):182-197

Farmani R, Butler D (2014) Implications of urban form on water distribution systems performance. Water Resour Manag 28:83-97

Fujiwara O, Tung H (1991) Reliability improvement for water distribution networks through increasing pipe size. Water Resour Res 27(7):1395-1402

Gargano R, Pianese D (2000) Reliability as tool for hydraulic network planning. J Hydraul Eng 126(5):354-364

Germanopoulos G (1985) A technical note on the inclusion of pressure dependent demand and leakage terms in water supply network models. Civ Eng Syst 2(3):171-179

Gheisi A, Naser G (2013) On the significance of maximum number of component failures in reliability analysis of water distribution systems. Urban Water J 10(1):10-25

Gheisi A, Naser G (2015) Multistate reliability of water-distribution systems: comparison of surrogate measures. J Water Resour Plann Manag. doi:10.1061/(ASCE)WR.1943-5452.0000529

Greco R, Di Nardo A, Santonastaso G (2012) Resilience and entropy as indices of robustness of water distribution networks. J Hydroinf 14(3):761-771

Gupta R, Bhave PR (1996) Comparison of methods for predicting deficient network performance. J Water Resour Plan Manag 122(3):214-217

Jayaram N, Srinivasan K (2008) Performance-based optimal design and rehabilitation of water distribution networks using life cycle costing. Water Resour Res 44, W01417

Kalungi P, Tanyimboh TT (2003) Redundancy model for water distribution systems. Reliab Eng Syst Saf 82(3):275-286

Khomsi D, Walters GA, Thorley ARD, Ouazar D (1996) Reliability tester for water distribution networks. J Comput Civil Eng 10(1):10-19 
Kovalenko Y, Gorev NB, Kodzhespirova IF, Prokhorov E et al (2014) Convergence of a hydraulic solver with pressure-dependent demands. Water Resour Manag 28:1013-1031

Liu H, Savić DA, Kapelan Z, Zhao M, Yuan Y, Zhao H (2014) A diameter-sensitive flow entropy method for reliability consideration in water distribution system design. Water Resour Res 50(7):5597-5610

Prasad TD, Park NS (2004) Multiobjective genetic algorithms for design of water distribution networks. J Water Resour Plan Manag 130(1):73-82

Raad DN, Sinske AN, van Vuuren JH (2010) Comparison of four reliability surrogate measures for water distribution systems design. Water Resour Res 46(5), W05524

Reca J, Martinez J, Banos R, Gil C (2008) Optimal design of gravity-fed looped water distribution networks considering the resilience index. J Water Resour Plan Manag 134(3):234-238

Rossman LA (2002) EPANET 2 user's manual. Water Supply and Water Resources Division, National Risk Management Research Laboratory, Cincinnati

Rowell WF, Barnes J (1982) Obtaining layout of water distribution systems. J Hydraul Div 108(1):137-148

Saleh SHA, Tanyimboh TT (2014) Optimal design of water distribution systems based on entropy and topology. Water Resour Manag 28(11):3555-3575

Seyoum AG, Tanyimboh TT (2014) Pressure dependent network water quality modeling. J Water Manag 167(6): $342-355$

Seyoum AG, Tanyimboh TT, Siew C (2013) Assessment of water quality modelling capabilities of EPANET multi-species and pressure dependent extension models. Water Sci Technol Water Supply 13(4):1161-1166

Shannon CE (1948) A mathematical theory of communication. Bell Syst Tech J 27:379-423 and 623-656

Shirzad A, Tabesh M, Farmani R, Mohammadi M (2013) Pressure-discharge relations with application to head-driven simulation of water distribution networks. J Water Resour Plan Manag 139(6):660-670

Siew C, Tanyimboh TT (2012a) Pressure-dependent EPANET extension. Water Resour Manag 26(6):1477-1498

Siew C, Tanyimboh TT (2012b) Penalty-free feasibility boundary-convergent multi-objective evolutionary algorithm for the optimization of water distribution systems. Water Resour Manag 26(15):4485-4507

Siew C, Tanyimboh T, Seyoum A (2014) Assessment of penalty-free multi-objective evolutionary optimization approach for the design and rehabilitation of water distribution systems. Water Resour Manage 28(2):373-389

Singh VP, Oh J (2015) A Tsallis entropy-based redundancy measure for water distribution networks. Phys A 421: 360-376

Su Y, Mays LW, Duan N, Lansey KE (1987) Reliability based optimization model for water distribution systems. J Hydraul Eng 114(12):1539-1556

Tabesh M, Soltani J, Farmani R, Savic DA (2009) Assessing pipe failure rate and mechanical reliability of water distribution networks using data driven modeling. J Hydroinf 11(1):1-17

Tanyimboh TT (1993) An entropy-based approach to the optimum design of reliable water distribution networks. $\mathrm{PhD}$ thesis, University of Liverpool, p 221

Tanyimboh TT, Kalungi P (2008) Optimal long term design, rehabilitation and upgrading of water distribution networks. Eng Optim 40(7):637-654

Tanyimboh TT, Kalungi P (2009) Multicriteria assessment of optimal design, rehabilitation and upgrading schemes for water distribution networks. Civ Eng Environ Syst 26(2):117-140

Tanyimboh TT, Setiadi Y (2008) Sensitivity analysis of entropy-constrained designs of water distribution systems. Eng Optim 40(5):439-457

Tanyimboh TT, Sheahan C (2002) A maximum entropy based approach to the layout optimization of water distribution systems. Civ Eng Environ Syst 19(3):223-253

Tanyimboh TT, Tabesh M (1997) Discussion: comparison of methods for predicting deficient-network performance. J Water Resour Plan Manag 123(6):369-370. doi:10.1061/(ASCE)0733-9496(1997)123:6(369)

Tanyimboh TT, Templeman AB (1993a) Calculating maximum entropy flows in networks. J Oper Res Soc 44(4): 383-396

Tanyimboh TT, Templeman AB (1993b) Optimum design of flexible water distribution networks. Civ Eng Syst 10(3):243-258

Tanyimboh TT, Templeman AB (1993c) Maximum entropy flows for single-source networks. Eng Optim 22(1): 49-63

Tanyimboh TT, Templeman AB (1993d) Using entropy in water distribution networks. In: Coulbeck B (ed) Integrated computer applications in water supply, Volume 1. Research Studies Press Ltd, Taunton, England, ISBN 086380154 4, pp 77-90

Tanyimboh TT, Templeman AB (1995) A new method for calculating the reliability of single-source networks. 6th International Conference on Computing in Civil and Structural Engineering, Cambridge, UK, 28-30 August 1995, pp 1-9

Tanyimboh TT, Templeman AB (1998) Calculating the reliability of single source networks by source head method. Adv Eng Softw 29(7-9):449-505 
Tanyimboh T, Templeman A (2000) A quantified assessment of the relationship between the reliability and entropy of water distribution systems. Eng Optim 33(2):179-199

Tanyimboh TT, Templeman AB (2010) Seamless pressure-deficient water distribution system model. J Water Manag 163(8):389-396

Tanyimboh TT, Burd R, Burrows R, Tabesh M (1999) Modelling and reliability analysis of water distribution systems. Water Sci Technol 39(4):249-255

Tanyimboh TT, Tabesh M, Burrows R (2001) An appraisal of source head methods for calculating the reliability of water distribution networks. J Water Resour Plan Manag 127(4):206-213

Tanyimboh TT, Tahar B, Templeman AB (2003) Pressure-driven modelling of water distribution systems. Water Sci Technol Water Supply 3(1-2):255-261

Tanyimboh TT, Tietavainen MT, Saleh SAE (2011) Reliability assessment of water distribution systems with statistical entropy and other surrogate measures. Water Sci Technol Water Supply 11(4):437-443

Templeman AB (1982) Discussion of optimization of looped water distribution systems, by GE Quindry, ED Brill and JC Liebman. J Environ Eng 108(3):599-602

Todini E (2000) Looped water distribution networks design using a resilience index based heuristic approach. Urban Water 2(3):115-122

Tsallis C (1988) Possible generalization of Boltzmann-Gibbs statistics. J Stat Phys 52(1-2):479-487

Twort AC, Ratnayaka DD, Brandt MJ (2000) Water supply. Arnold, London

Vaabel J, Ainola L, Koppel T (2006) Hydraulic power analysis for determination of characteristics of a water distribution system. 8th Annual Water Distribution Systems Analysis Symposium, ASCE, Reston, VA, USA

Wagner JM, Shamir U, Marks DH (1988) Water distribution reliability. J Water Resour Plan Manag 114(3):253294

Wu W, Maier HR, Simpson AR (2011) Surplus power factor as a resilience measure for assessing hydraulic reliability in water transmission system optimization. J Water Resour Plan Manag 137(6):542-546 